\title{
Functional Time Series Models and the APC Models: A Comparative Study on the Lung Cancer Incidence Rates in Denmark
}

\author{
Farah Yasmeen and Saba Mughal \\ Department of Statistics, University of Karachi, Karachi 75270, Pakistan
}

\begin{abstract}
Analysis, precise interpretation and forecasting of incidence and mortality rates are very important aspects in epidemiology and demography. The purpose of this study is to apply two different methodologies, namely the FTS (functional time series) models and APC (age-period-cohort) models on a single data set. We intend to compare the results obtained and to see the performances of these two approaches. We used FTS models on age-specific incidence rates taking age as a continuous function that is varying by time. Here we examined the age variations by using FPC (functional principal component) analysis. We also obtained the forecast of the entire incidence curve. Then we applied the APC models on the same data where we explored the age, period and cohort effects separately. We illustrated these approaches by using lung cancer incidence rates for males in Denmark, obtained from R-package "Epi” available on CRAN (Comprehensive R Archive Network). It was found that there was a rapid increase in lung cancer incidence rates in Denmark since 1960, and the highest rates were seen in the year 1985. After that, the rates started to level off. A continuous increment was also found in incidence rates since 1958 birth cohorts. These rates stabilized in 1905 and started decreasing since 1925 . The first four basis functions of the FTS model explained about $98.5 \%, 1.2 \%, 0.2 \%$, and $0.1 \%$ of the total variation, respectively. We also obtained 20-year predictions and suggested that future trends for the male lung cancer incidence rates in Denmark will decrease in all ages.
\end{abstract}

Key words: Functional time series models, forecast, age-period-cohort models, age-specific incidence rates, lung cancer.

\section{Introduction}

FDA (functional data analysis) is a branch of statistics that analyzes data to provide information about curve or surfaces, instead of single observation. These types of data sets are referred as functional data. In FTS (functional time series), the data provide information about curves (which are considered as unit of observations) observed in regular intervals in time. The FTS models were first introduced by Hyndman et al. [1], where those models were used in forecasting a series of functional data observed over time. The functions were observed, with error at time $t=1, \ldots, n$, and the forecasts were obtained for time $t=n+$

Corresponding author: Farah Yasmeen, Ph.D., assistant professor, research fields: biostatistics, demography, multivariate and time series analysis. E-mail: riazfarah@yahoo.com.
$1, \ldots, n+h$. For example, in demography, the mortality or fertility rates can be modeled taking age as a continuous function in time " $t$ ". The model is a generalization of the LC (Lee-Carter) model commonly used in mortality and fertility forecasting. Lee et al. [2] proposed a methodology for modeling and extrapolating trends in mortality rates having long term periods. There were many applications involving functional time series including those studied in Erbas et al. [3, 4]. Also, these models were applied in forecasting the mortality rates of chronic diseases such as breast cancer by Yasmeen et al. [5, 6].

The APC (age-period-cohort) models, one of the most commonly used statistical methods, were used to analyze trends for the mortality/incidence rates in demography and epidemiology [7]. These rates were observed over a long time period and for a broad age 
range, that were categorized by age (at death/at follow up), the period (year of death/date of follow up) and the cohort (date of birth). The purpose of designing APC models was to estimate the effects of three variables including age, period and cohort [8]. The age effect could be determined by the differing risks correspond to different age groups, the period effect indicated a change in rate which is associated with all age groups like a change in treatment that would reduce mortality rate in all ages, and the cohort effects were associated with long-term exposures or long-term habits whereby different generations were exposed to different risks. Period and cohort effects explained the changes in the rates associated with time.

The major problem with APC models was an exact linear dependency among the three factors, as discussed by Holford [8]. The information of any two variables provides information about the third variable as " $c=a-p$ ", where " $c$ " represents the cohort effect and " $a$ " and " $p$ " are the age and period effects, respectively. In APC model, the effect of the three variables becomes non-linear on the log rate scale and hence, the modeling of the rates and parameterization has to be done carefully. The identifiability problem was well known, and a number of solutions were proposed in the past. The first paper that showed the effects by using different arbitrary constraints on the period and cohort parameters so that one could ensure the problem of identifiability was proposed by Holford [9]. To deal with the identification problem, standard procedures proposed by Clayton et al. $[10,11]$ were also used, where the mortality rate was decomposed into a common linear trend (drift), a non-linear period effect and a non-linear cohort effect. More recently, a method was proposed by Carstensen [12], where the authors suggested no constraint on age parameter. They suggested selecting a reference point for either the period or the cohort term and constraining the other factor to be zero on average. This method was also used by Su et al. [13] to check the impact of a new screening program on the mortality rates in cervical cancer in Taiwan for the two different time periods.

The methodologies discussed above were widely used in the field of demography and epidemiology, and both of the methods have their own importance in these fields. In this paper, we intend to compare the results obtained and to check the performance of these two approaches. To the best of our knowledge, this is the first study to show the performance of both APC and FTS models on the same data set, and to discuss their advantages and limitations.

\section{Material and Methods}

\subsection{Statistical Data}

For illustration, we consider the data on the lung cancer incidence rates for males in Denmark from 1943-1995. The data were obtained from R-package "Epi" [14]. The source of the data for this package is the Danish cancer registry and statistics, Denmark. The incidence rates were available in the ages 40-89 years, where cases and person-years were tabulated by age, the date of diagnosis (period) and the date of birth (cohort) in 5-year classes.

\subsection{Fitting of FTS (Functional Time Series) Models}

As described earlier, Hyndman et al. [1] proposed an FTS model on log fertility/mortality rates and obtained the future curves, where each curve was considered as a unit of analysis rather than discrete observations. Here we applied this model on the log incidence rates. The main model is given by

$$
y_{t}\left(x_{i}\right)=f_{t}\left(x_{i}\right)+\sigma_{t}\left(x_{i}\right) \varepsilon_{t, i}
$$

where, $y_{t}\left(x_{i}\right)$ is the log incidence rates at age ' $x$ ' in year ' $t$ ', $i=1, \ldots, p, f_{t}\left(x_{i}\right)$ is the smooth function of $x_{i}, \sigma_{t}\left(x_{i}\right)$ allows heteroscedasticity in $x_{i}$, and $\varepsilon_{t, i}$ is independent and identically distributed standard normal random variable.

The first step was to estimate smooth functions by using non-parametric smoothing in order to reduce the randomness of the observed data. Then the smooth curves were decomposed into basis functions and their 
corresponding coefficients by using functional principle component analysis. Following model was used for functional principal component decomposition

$$
f_{t}(x)=\mu(x)+\sum_{k=1}^{K} \beta_{t, k} \phi_{k}(x)+e_{t}(x)
$$

where, $\mu(x)$ is a measure of location of log incidence rates over ' $t$ ', $\phi_{k}(x)$ is a set of orthogonal basis functions, $\beta_{t, k}$ is the univariate time series for each $k=1, \ldots, K$ and $e_{t}(x)$ is independent and identically distributed random functions with zero mean and they are assumed to be serially uncorrelated.

To forecast the time series cofficients, we used univariate time series models. The h-step ahead forecast of the entire incidence curve can be obtained by multiplying these foracasts of individual coefficients with the estimated basis functions and summing the results.

$$
\hat{y}_{n+h}(x)=\hat{\mu}(x)+\sum_{k=1}^{K} \hat{\beta}_{n+h, k} \hat{\phi}_{k}(x)
$$

where, $\hat{\mu}(x)$ and $\hat{\phi}_{k}(x)$ are the estimates of mean function and basis functions, respectively, $\hat{\beta}_{k, n+h}$ denotes the h-step ahead forecast of $\beta_{t, k}$ coefficients. Forecast variance and the prediction intervals for the h-step forecaste curves can also be determined by the proposed method [1]. All options for model fitting and forecasting are available in the "demography" package [15] and "forecast" package [16] for R, available on CRAN (Comprehensive R Archive Network).

\subsection{Fitting of APC (Age-Period-Cohort) Models}

The APC models are used to investigate the age, period and cohort effects independently. However, these effects cannot be analyzed simultaneously, as the model suffers a problem of identifiability. The classical model to estimate the age and cohort effects is given by

$$
\rho_{a c}=\alpha_{a}^{\prime} \gamma_{c}^{\prime}
$$

This is the age-cohort model, where, $\rho_{a c}$ are the observed rates at age ' $a$ ' for the cohort ' $c$ ', parameter $\alpha_{a}^{\prime}$ represents the age-specific rates and parameter $\gamma_{c}^{\prime}$ shows their dependence upon cohort. If we fix one of the $\gamma_{c}$ equal to zero, then the antilog $\left(\gamma_{c}^{\prime}\right)$ is the relative risk of every single cohort that is relative to the reference cohort and $\alpha_{a}^{\prime}$ is the fitted age-specific rates for this reference cohort.

Another model is the age-period model given by

$$
\rho_{a p}=\alpha_{a}^{\prime} \beta_{p}^{\prime}
$$

where, $\rho_{a p}$ is the observed rates at age ' $a$ ' in period ' $p$ ', the parameter $\alpha_{a}^{\prime}$ represents the age-specific rates and parameter $\beta_{p}^{\prime}$ shows their dependence upon period.

Finally, the age-period-cohort model can be expressed as

$$
Y_{a p}=\alpha_{a}+\delta_{p}\left(p-p_{o}\right)+\delta_{c}\left(c-c_{o}\right)
$$

where, $Y_{a p}$ is the log rates at age ' $a$ ' in period ' $p$ ' for the cohort ' $c=p-a$ ', the parameter $\alpha_{a}$ represents relationship between age and incidence, $\delta_{p}$ and $\delta_{c}$ represent (constant) change in log-rates between adjacent period and cohort, and $p_{o}$ and $c_{o}$ are the reference period and cohort, respectively. By taking antilog of the above expression, we can write

$$
\rho_{a p}=\alpha_{a}^{\prime}\left(\delta_{p}^{\prime}\right)^{\left(p-p_{o}\right)}\left(\delta_{c}^{\prime}\right)^{\left(c-c_{o}\right)}
$$

where, $\delta_{p}^{\prime}$ and $\delta_{c}^{\prime}$ are the relative risks between adjacent period and cohort, respectively.

Recently, Carstensen [12] proposed a methodology that consists of two mathematical steps. First step was to model the age effect and the cohort effect by choosing a suitable reference for the cohort. In this way, the age-effects would be the log rates for the reference cohort and the cohort effects would be the log RRs (relative risks). The functions $\hat{f}(a)$ and $\hat{h}(c)$ were used as age and cohort effects, respectively. The results were used as offset variable in the following model with period effects:

$$
\log [\lambda(a, p)]=[\hat{f}(a)+\hat{h}(c)]+g(p)
$$

In this way, the period effects would be the residuals of log relative risk. This small trick was used for model fitting (parameterization) where natural spline functions were used to smooth rates for modeling. All options for model fitting used are available in the "Epi” package [14] of R, available on CRAN. 


\section{Results and Analysis}

\subsection{Results of FTS (Functional Time Series) Models}

In Fig. 1, the male lung cancer incidence rates of Denmark were plotted as functional time series. The curves were presented in rainbow order where the earlier years were plotted in red and the recent years in violet. Each curve was representing a group of 5-year. Here we can see that incidence rates were low in earliest years (from 1943) but higher in recent year (1995) for almost all ages. There was a rapid increase in incidence rates from the age of 55 followed by a decline from 78 years of age. There was a high risk found in the ages of 70's for lung cancer incidence in Denmark.

From the original series of incidence rates, the smooth rates were estimated using weighted regression B-spline with a concavity constraint. We used functional regression model in order to obtain an adequate fit to the data. We obtained the first four basis functions with their time series coefficients. These basis functions explained $98.5 \%, 1.2 \%, 0.2 \%$, and $0.1 \%$ of the total variation.

Fig. 2 depicts the overall mean function, first four estimated basis functions and their coefficients with 80\% prediction intervals. The basis functions modeled incidence rates in different age groups. The first basis function $\emptyset_{1}(x)$ showed an increase in lung cancer incidence for ages around 70 years, where $\emptyset_{2}(x)$ showed a contrast those between ages 40 years and 60 years. While their respective coefficients showed a decline in future years. The other basis functions $\emptyset_{3}(x)$ and $\emptyset_{4}(x)$ were complex as they involved third and higher order effects, so we didn't attempt to interpret them.

Fig. 3 shows the twenty-year forecasts for the male lung cancer incidence from 1996 to 2015. These forecasts were obtained by multiplying the forecast values of coefficients with the estimated basis functions and summing the results. The grey curves show the original smoothed log incidence rates from 1943 to 1995 . It shows that there will be a decline in the incidence rates for almost all ages. A slower decline is expected in the ages of 40-50 years and a relatively larger decline for the ages 50-55 years. It is clear from Fig. 3 that the future lung cancer incidence rates are expected to decrease very fast for older ages (60 years and above).

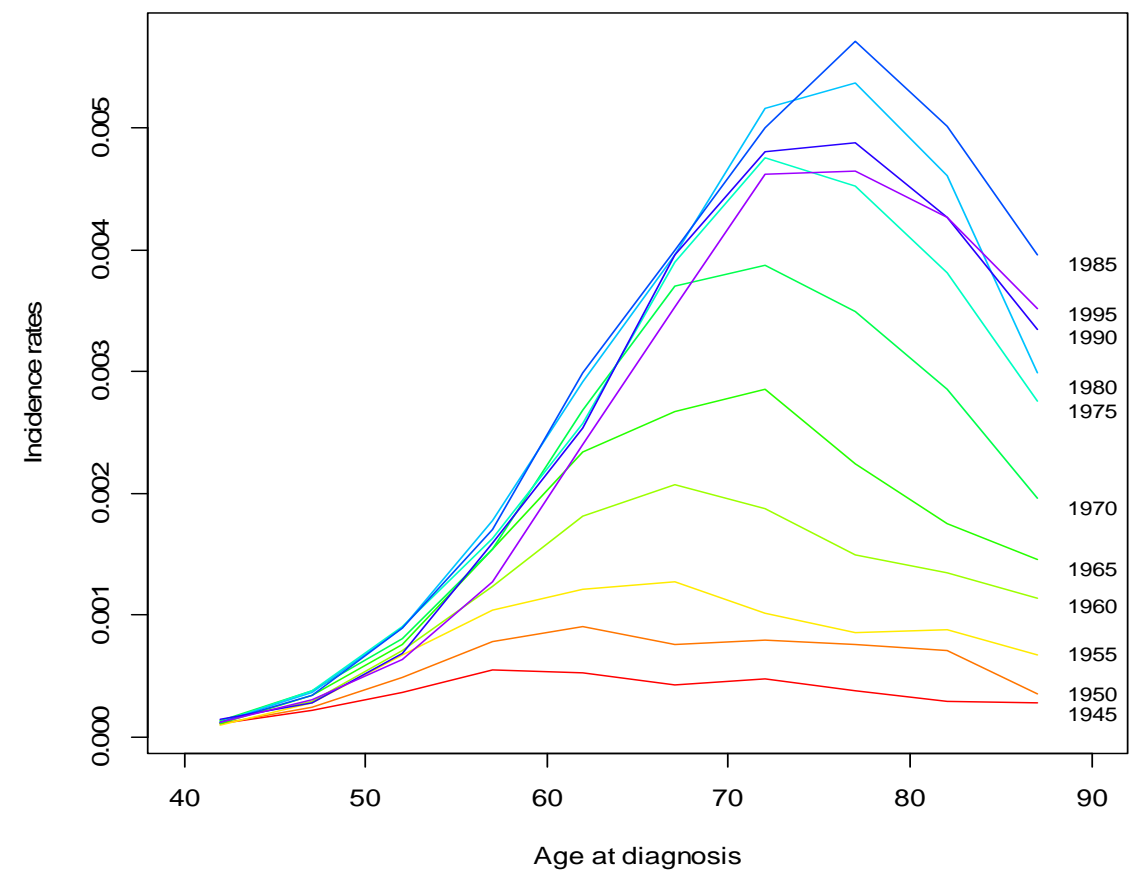

Fig. 1 Lung cancer incidence rates in Denmark (1945-1995). 
Functional Time Series Models and the APC Models: A Comparative Study on the Lung Cancer Incidence Rates in Denmark
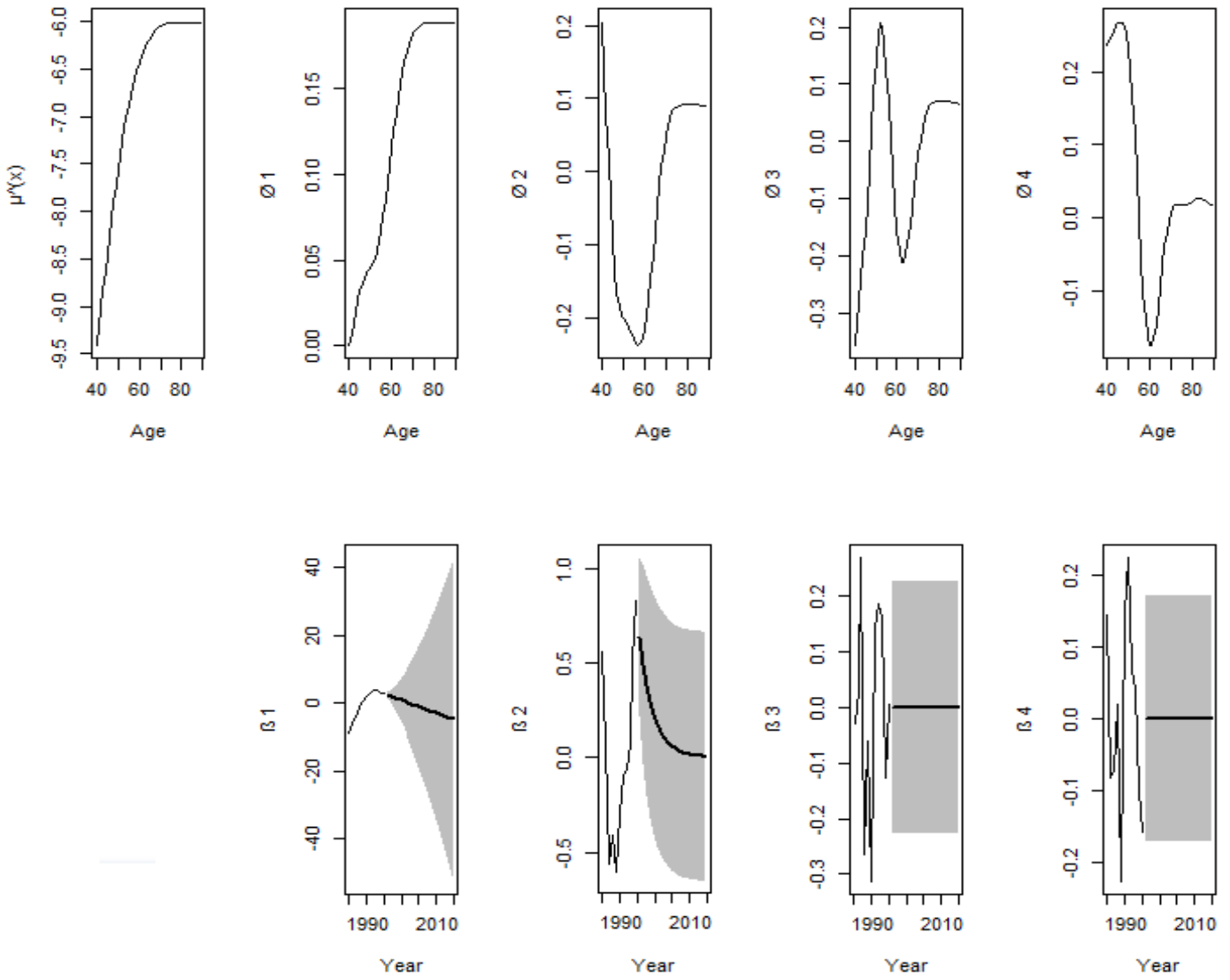

Fig. 2 The first four basis functions and forecasts of their coefficients.

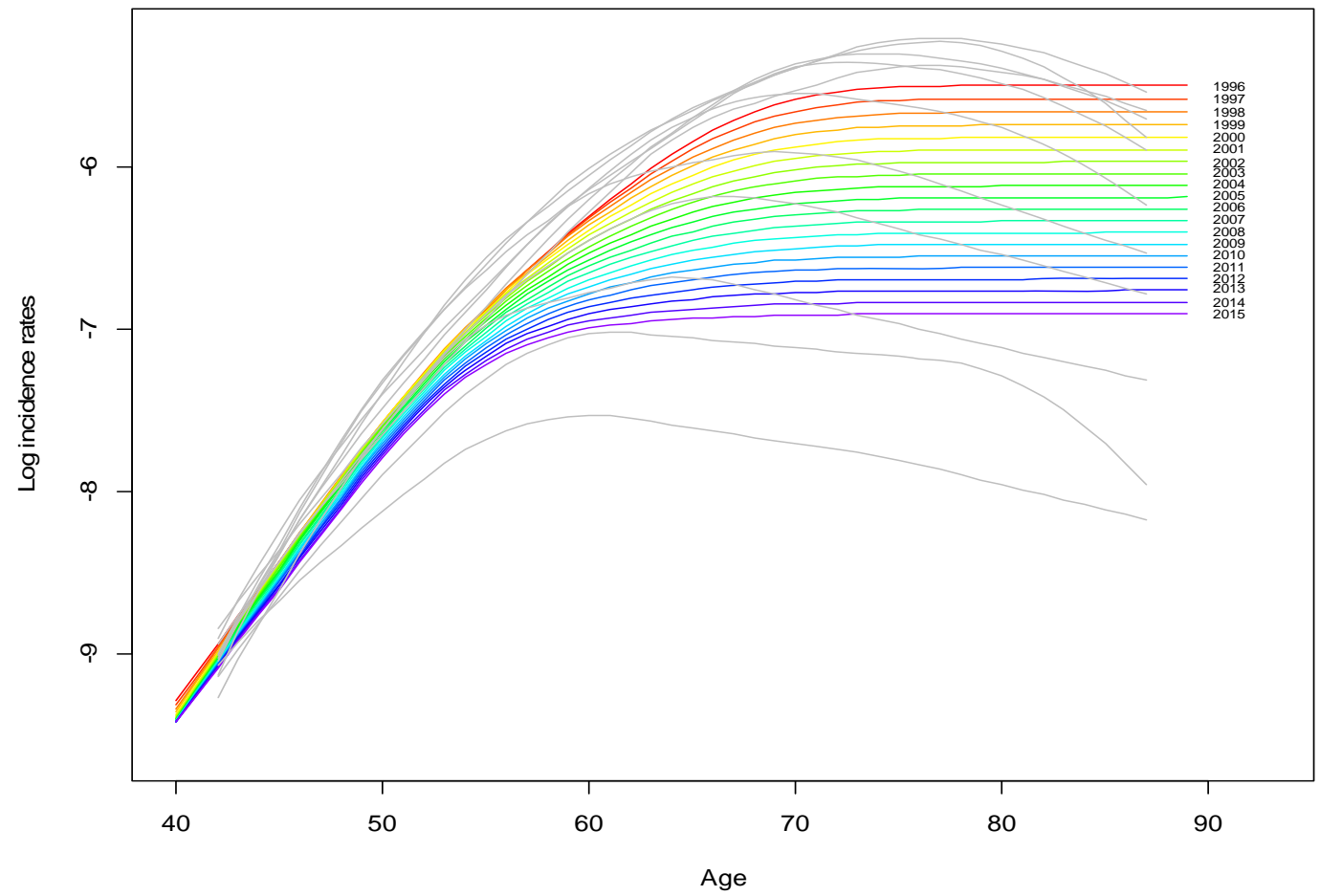

Fig. 3 20-year forecasts (1996-2015) of log incidence rates in Denmark. The original log incidence rates are plotted with grey curves in the background. 


\subsection{Results of APC (Age-Period-Cohort) Models}

Fig. 4 shows incidence rates of lung cancer in Denmark for males, where each curve in this graph represents a longitudinal series of rates. It depicts the evolution of incidence within a birth cohort against age. Here we can see that the rates for earlier birth cohorts were relatively low but birth cohorts for the middle years showed a clear increment in the incidence rates, especially for the ages of 60-75 years.

Finally the age, period and cohort effects were estimated by using regression model based on Poisson distribution. Fig. 5 shows the estimated effects of age at diagnosis on the age scale and the two other curves showing cohort and period effects. The right most curve, which is the shortest one, shows the period effect whereas the middle curve shows the cohort effect on the calendar time scale with $95 \%$ confidence intervals. The vertical scales show rate-scale for the age-effects and the relative risk-scale for the period and cohort effects. Here we used the sequential approach by first fitting the age-cohort model and then the period model to the residuals.

The reference cohort can be found at $\left(c_{o}, 1\right)$ where $c_{o}=1,900$, the corresponding value at the rate ratio scale is one. The age-effect refers to the reference cohort. The age-curve (the left most curve of Fig. 5) shows an increasing trend from age 40 through 70 years. For the age-group 70-80 years, the highest incidence rates were found followed by a small decline after 80 years. The cohort effect can be read as the RRs relative to the reference cohort. There is a clear tendency that cohorts born earlier (before 1900) showed relatively lower incidence rates and for those who born later, relatively higher rates were observed. For the birth cohorts of 1900-1910, the rates were stable but in the cohort of 1920, the highest incidence rates were found and they level off after that. In contrast, the curve of period-effect shows increasing incidence rates since 1945 till 1980, followed by a decline after 1980.

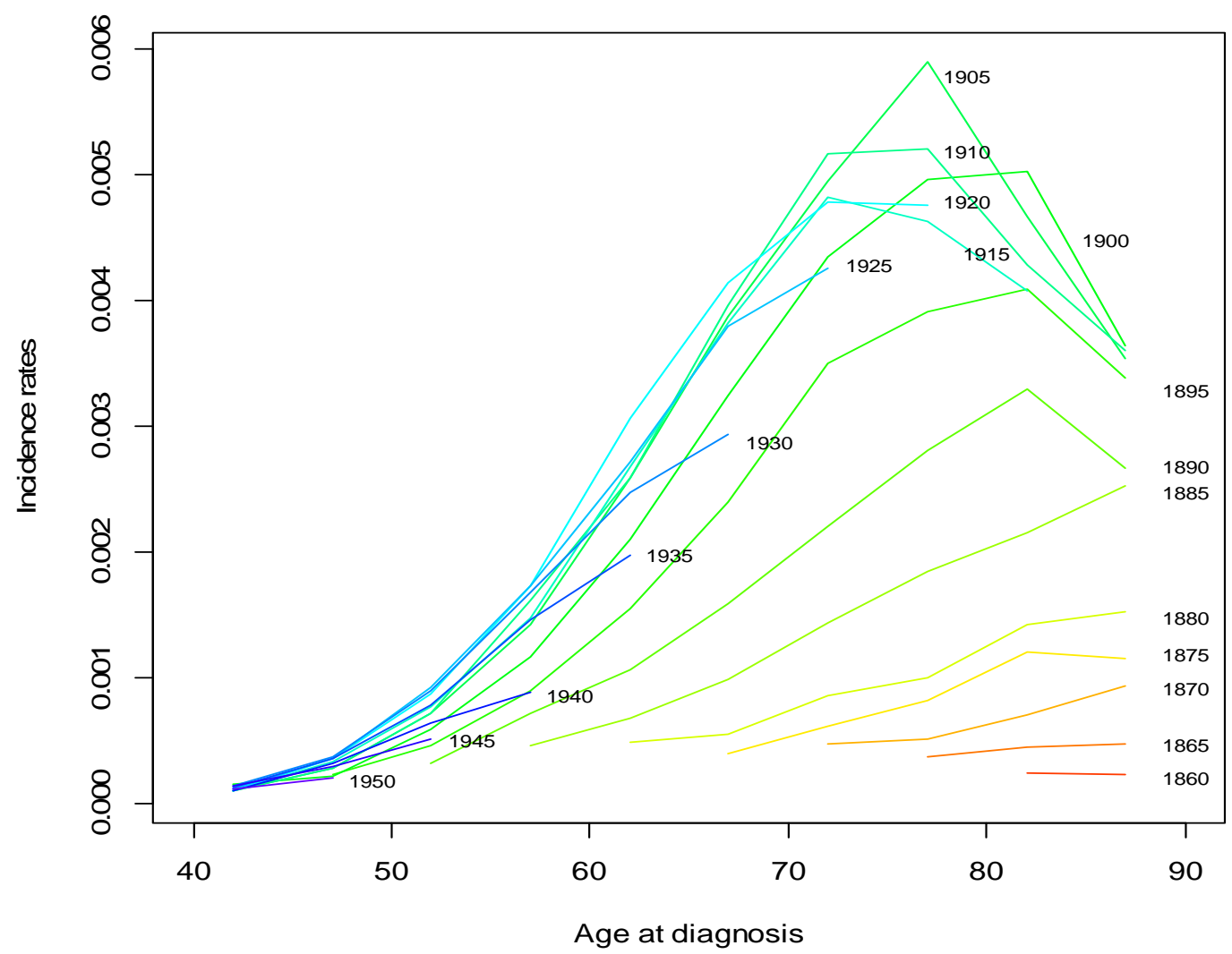

Fig. 4 Lung cancer incidence rates in Denmark (Cohort effects). 


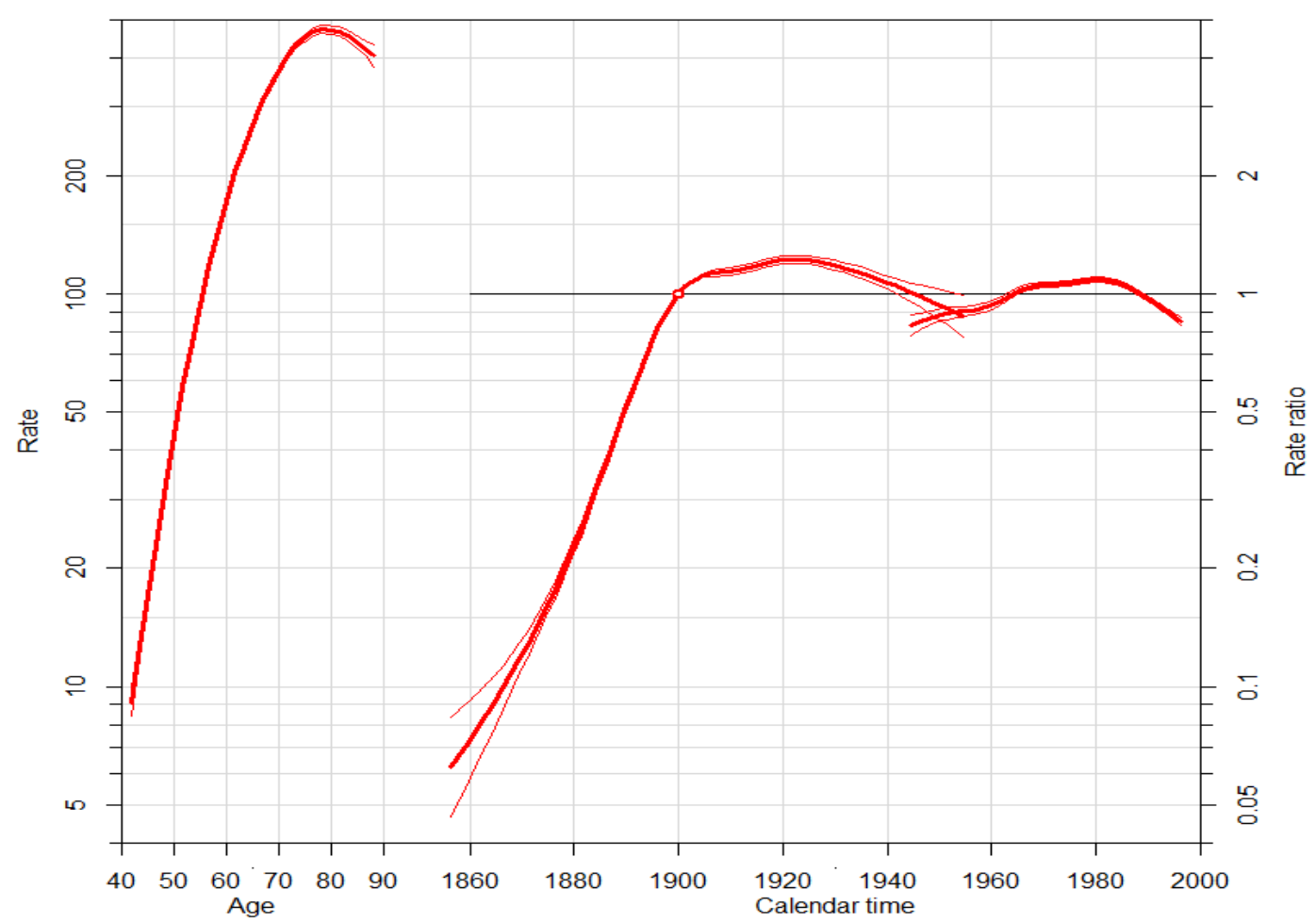

Fig. 5 The age, period and cohort effects from APC model for the male lung cancer incidence data of Denmark.

\section{Strength and Limitations}

Every model has its own advantages and limitations. FTS model deals with the curves where we can analyze subtle variations among years. The age functions can be analyzed in detail with their associated time series coefficients as the FTS model decomposes curves via basis function expansion. It also gives forecast results better than any other approaches. But this model does not incorporate the cohort term in the model. Cohort effect has a strong impact on the causes of the chronic diseases where we require prolong exposures.

On the other hand, the APC models are the commonly used statistical method which incorporate all three effects of age, period (year of death/diagnose) and cohort (year of birth). They give better understanding by providing the estimates of these factors and one can also extrapolate the future rates using these models. But the parameter identification problem is considered as a unique and unfortunate limitation of an APC model. For this reason, the predictions also depend on the parameterization chosen and long term predictions are notoriously unstable. At any future point, the changes in the parameterization will cancel out the predictions as the model is not uniquely parameterized.

\section{Conclusions}

In this study, we applied the FTS models to the data of lung cancer incidence rates in Denmark. We also obtained the twenty-year forecasts of the incidence curves. The future incidence rates are expected to decline for all ages. The rate of decline will be faster for older ages (60 years and above) and relatively slower for the age-group 40-50 years.

These methodologies will be useful especially in the field of epidemiology. By using FTS models, we can obtain the forecasts for the mortality/incidence due to any chronic disease. These forecasts will be very helpful in building public health policies, implementing screening programs and treatments for the disease prevention.

We also applied the APC models on the same data set, where we fitted the model sequentially in order to 
get estimates for the age, period and cohort effects. We found significant results for the period and cohort effects in lung cancer incidence trends. In contrast to the FTS models, the APC models can be used if one aims to capture all the three effects that play important roles in the medium of time trends in disease incidence/mortality rates. As in case of mortality, the period effect counts a lot if sudden changes occur such as implementation of new screening program. Similarly for some chronic diseases, certain generations or cohorts have greater exposure where long-term habits affect the disease rates. In these cases, the use of APC models is supposed to be more appropriate.

\section{References}

[1] Hyndman, R. J., and Ullah, M. S. 2007. "Robust Forecasting of Mortality and Fertility Rates: A Functional Data Approach.” Comput. Stat. Data Anal. 51 (10): 4942-56.

[2] Lee, R. D., and Carter, L. R. 1992. "Modeling and Forecasting US Mortality." Journal of the American Statistical Association 87 (419): 659-71.

[3] Erbas, B., Hyndman, R. J., and Gertig, D. M. 2007. "Forecasting Age-Specific Breast Cancer Mortality Using Functional Data Analysis.” Stat. Med. 26 (2): 458-70.

[4] Erbas, B., Ullah, S., Hyndman, R. J., Scollo, M., and Abramson, M. 2012. "Forecasts of COPD Mortality in Australia: 2006-2025.” BMC Medical Research Methodology 12: 17. Accessed August 6, 2014. http://www.biomedcentral.com/1471-2288/12/17 .

[5] Yasmeen, F., Hyndman, R. J., and Erbas, B. 2010. "Forecasting Age-Related Changes in Breast Cancer Mortality among White and Black US Women: A Functional Data Approach.” Cancer Epidemiology 34 (5): 542-9.

[6] Yasmeen, F., and Zaheer, S. 2014. "Functional Time
Series Models to Estimate Future Age-Specific Breast Cancer Incidence Rates for Women in Karachi, Pakistan.” Journal of Health Science 2 (5): 213-21.

[7] Jatoi, I., Anderson, W. F., Rao, S. R., and Devesa, S. S. 2005. "Breast Cancer Trends among Black and White Women in the United States." Journal of Clinical Oncology 23 (31): 7836-41.

[8] Holford, T. R. 1992. "Analysing the Temporal Effects of Age, Period and Cohort.” Statistical Methods in Medical Research 1 (3): 317-37.

[9] Holford, T. R. 1983. "The Estimation of Age, Period and Cohort Effects for Vital Rates.” Biometrics 39 (2): 311-24.

[10] Clayton, D., and Schifflers, E. 1987. "Models for Temporal Variation in Cancer Rates. I: Age-Period and Age-Cohort Models.” Statistics in Medicine 6 (4): 449-67.

[11] Clayton, D., and Schifflers, E. 1987. "Models for Temporal Variation in Cancer Rates. II: Age-Period-Cohort Models.” Statistics in Medicine 6 (4): 469-81.

[12] Carstensen, B. 2007. "Age-Period-Cohort Models for the Lexis Diagram.” Statistics in Medicine 26 (15): 3018-45.

[13] Su, S. Y., Huang, J. Y., Ho, C. C., and Liaw, Y. P. 2013. "Evidence for Cervical Cancer Mortality with Screening Program in Taiwan, 1981-2010: Age-Period-Cohort Model.” BMC Public Health 13. Accessed August 6, 2014. www.biomedcentral.com/1471-2458/13/13.

[14] Carstensen, B., Plummer, M., Laara, E., and Hills, M. 2014. Epi: A Package for Epidemiological Analysis in R, version 1.1.65. Accessed August 6, 2014. www.bendixcarstensen.com/ Epi/.

[15] Hyndman, R. J. 2010. Demography: Forecasting Mortality, Fertility, Migration and Population Data, R Package, Version 1.07, with Contribution from Heather Booth, Leonie Tickle and John Maindonald. Accessed August 6, 2014. Www. robjhyndman.com/software/demography.

[16] Hyndman, R. J. 2014. Forecast: Forecasting Functions of Time Series Models, R Package, Version 5.3. Accessed August 6, 2014. www.robjhyndman.com/software/forecast. 